\title{
Migratory Loose Bodies from the Ankle Joint into the Flexor Hallucis Longus Tendon Sheath
}

\author{
Authors: \\ *Jenn Shiunn Wong, PNM Tyrrell, B Tins, T Woo, N Winn, *VN Cassar- \\ Pullicino \\ 1. Department of Radiology, Robert Jones and Agnes Hunt Orthopaedic Hospital, \\ NHS Foundation Trust, Owestry, UK \\ *Correspondence to victor.pullicino@nhs.net \\ Disclosure: $\quad$ The authors have declared no conflicts of interest. \\ Received: $\quad 12.11 .20$ \\ Accepted: $\quad 10.09 .21$ \\ Keywords: $\quad$ Ankle joint, flexor hallucis longus (FHL) tendon sheath, loose bodies, migratory, \\ osteoarthritis. \\ Citation: $\quad$ EMJ Radiol. 2021; DOl/10.33590/emjradiol/20-002571.
}

\begin{abstract}
Objective: Loose bodies resulting from any form of osteochondral insult can migrate out of their intra-articular position to adjacent compartments. This retrospective study aims to illustrate the phenomenon of loose bodies migration from the ankle joint into the flexor hallucis longus (FHL) tendon sheath.
\end{abstract}

Materials and Methods: Cases of loose bodies in the FHL tendon sheath were identified from the authors' radiological database by way of keyword interrogation, covering the modalities of CT, MRI, and ultrasound over a period of 11 years. The imaging features of the loose bodies were recorded, together with the presence of ankle instability and osteoarthritis. Patient demographics and relevant history, including trauma and surgery, were collected.

Results: Thirty-four cases including 33 patients, with a total of 125 loose bodies in the FHL tendon sheath, were identified. There were 58 loose bodies (46.4\%) in Zone 1 of the FHL tendon sheath, 65 loose bodies (52\%) in Zone 2, and 2 loose bodies (1.6\%) in Zone 3. All patients had features of ankle osteoarthritis on imaging, 14 of which had imaging features of ankle instability, and 19 patients had previous ankle trauma.

Conclusion: Osteochondral loose bodies originating from the ankle joint can migrate into the FHL tendon sheath. It is important to recognise this phenomenon as a distinct entity, different from primary tenosynovial chondromatosis of the FHL tendon sheath, which may have a different surgical management and clinical outcome. Detection of FHL tendon sheath loose bodies should also prompt closer examination for articular disease in the ankle joint.

\section{INTRODUCTION}

Communication between the flexor hallucis longus (FHL) tendon sheath and the ankle joint is well-recognised and is reportedly present in

around $17 \%$ of individuals. ${ }^{1}$ However, migration of osteochondral loose bodies from the ankle joint into the $\mathrm{FHL}$ tendon sheath is not a well-recognised phenomenon, with only one case described in the radiology literature so far. ${ }^{2}$ 
The authors hypothesise that loose bodies in the FHL tendon sheath migrated from the ankle joint, and the authors conducted this study to show their origin.

\section{MATERIALS AND METHODS}

Cases of loose bodies in the FHL tendon sheath were identified from the authors' radiological database by interrogating the body of reports for the keywords of "loose body" or "loose bodies" and "flexor hallucis longus" or "FHL." The period of the past 11 years was queried, covering the modalities of CT, MRI, and ultrasound.

The reports were scrutinised to only identify the cases that specifically documented the presence of loose bodies in the FHL tendon sheath. All cases then had their relevant imaging reviewed by two musculoskeletal radiologists (1 year and 40 years' experience) in order to document the number, size (largest dimension), location, and imaging appearances of the loose bodies.

The locations of the loose bodies were grouped into Zone 1 (behind the ankle joint to the orifice underneath the sustentaculum tali), Zone 2 (from the tunnel underneath the sustentaculum tali to the knot of Henry), or Zone 3 (a segment of FHL distal to the master knot of Henry to the phalangeal insertion). ${ }^{3}$ In addition, to differentiate from intratendinous calcification, the loose bodies had to be surrounded by tenosynovial fluid or be seen to efface the edge of the tendon or myotendinous junction of the $\mathrm{FHL}$ to be considered eligible. Cases with CT images were also assessed in multiplanar reconstruction to confirm their location within the tendon sheath if required. Cases with ultrasound examination had to demonstrate, on the saved static images, the loose bodies surrounded by anechoic fluid adjacent to the FHL tendon. Presence of radiological evidence of osteoarthritis and instability (asymmetric joint space loss and increased tibiotalar tilt) of the ankle joint were also recorded. Clinical notes were assessed to check for any previous history of trauma or surgery to the ankle joint.

CT images had been acquired on Siemens 16-slice or 64-slice scanners. MRI images had been acquired on a Siemens 1.5T or 3T scanners ( $\mathrm{T} 1$ sagittal, $\mathrm{T} 1$ turbo inversion recovery magnitude sagittal, proton density with fat saturation [PD
FS] coronal, PD FS axial). If a magnetic resonance (MR) arthrogram was performed, the sequences obtained were T1 sagittal, T1 FS coronal, T1 FS axial, PD FS coronal, and PD FS axial. Ultrasound was performed using the ACUSON S2000 (Siemens Healthineers, Erlangen, Germany) system. This is a retrospective study and ethical approval was therefore not required.

\section{RESULTS}

Initial interrogation of the authors' radiological database returned 127 cases that met the keyword-search criteria. After manually going through the body of the reports, 36 cases were identified whereby loose bodies had been documented within the $\mathrm{FHL}$ tendon sheath at the time of reporting. The images were reviewed, and 2 cases were removed due to inability to adequately confirm the location of the loose bodies on CT and ultrasound respectively.

In the series of 34 cases ( 33 patients), the mean age of the patients was 56.9 years (range: 20-84). There were 8 female and 25 male patients. Thirteen of the cases were of the left ankle and 21 of the right ankle. In terms of modalities, there were 21 cases of loose bodies identified on MRI, 11 cases on CT, and 2 cases on ultrasound. Of the 21 cases of MRI, there were 3 cases of MR arthrography examination confirming the communication between the ankle joint and FHL tendon sheath. There were 2 further cases where there were large ankle effusion showing definite communication with the FHL tendon sheath.

There were, altogether, 125 loose bodies documented in the 34 cases. There were 58 Zone 1 loose bodies (mean: $6.1 \mathrm{~mm}$; median: $5.0 \mathrm{~mm}$; range: 1.0-20.0 mm), 65 Zone 2 loose bodies (mean: $5.0 \mathrm{~mm}$; median: $4.0 \mathrm{~mm}$; range: 1.0-13.0 mm), and 2 loose bodies in Zone 3 (mean: $3.5 \mathrm{~mm}$; median: $3.5 \mathrm{~mm}$; range: $3.0-4.0 \mathrm{~mm}$ ). There were 16 cases of loose bodies in only Zone 1, 11 cases of only Zone 2, and 6 cases with loose bodies in both Zones 1 and 2 . There was 1 case with loose bodies in all 3 Zones. Concurrent loose bodies in the ankle joints were also demonstrated in 21 patients.

All patients had radiographic features of ankle osteoarthritis, including 4 patients who already had ankle fusion or replacement, and a further 13 patients who subsequently underwent ankle 
fusion or replacement. There was radiographic evidence of ankle instability in 14 cases with asymmetric joint space loss and increased tibiotalar tilt, indicative of lateral ligament dysfunction. Nineteen cases had previous injuries, ranging from minor sprain to previous ankle fractures, including a patient who had previously had a microfracture for a large subchondral cyst following an osteochondral injury 8 years earlier. There were no cases of rheumatoid arthritis or septic arthritis. There was 1 patient with a history of diabetes, although no features of diabetic neuropathy. Review of clinical notes revealed that the patients' main presenting complaints were related to their ankle joints rather than specifically from these loose bodies. There were no occasions where loose bodies in the FHL tendon sheath were detected on clinical examination.

\section{DISCUSSION}

Intra-articular loose bodies can result as sequelae of a wide range of pathologies, including osteoarthritis, trauma, neuropathic arthropathy, and inflammation. Primary synovial chondromatosis is much rarer in comparison. Loose bodies can migrate out of their intra-articular location to adjacent compartments via naturally occurring communications. Whilst the authors' series had not radiologically demonstrated actual migration of loose bodies from the ankle joint with sequential or interval imaging, the majority of patients (61.8\%) had loose bodies in both the ankle joint and the $\mathrm{FHL}$ tendon sheath. Furthermore, migration between these two compartments has been reported in the literature. ${ }^{2}$ In addition, loose body migration between different compartments is well documented in other body areas, including the knee joint with popliteal cyst, hip joint with iliopsoas bursa, and glenohumeral joint with biceps tendon sheath. ${ }^{4-6}$ Synovial chondromatosis of the subacromial bursa causing rotator cuff tear has also been reported, allowing the loose bodies to migrate into the glenohumeral joint ${ }^{7}$.

The authors grouped the location of the FHL tendon sheath loose bodies into Zones 1, 2, and 3 as per Lui (Figure 1). This is clinically relevant should surgical intervention be considered, as these loose bodies have been reported to become symptomatic during weight-bearing. ${ }^{2}$ Zone 1 tendoscopy is performed via posteromedial and posterolateral portals; Zone 2 is examined through posteromedial and plantar portals; and Zone 3 is examined through plantar toe portals. ${ }^{3}$

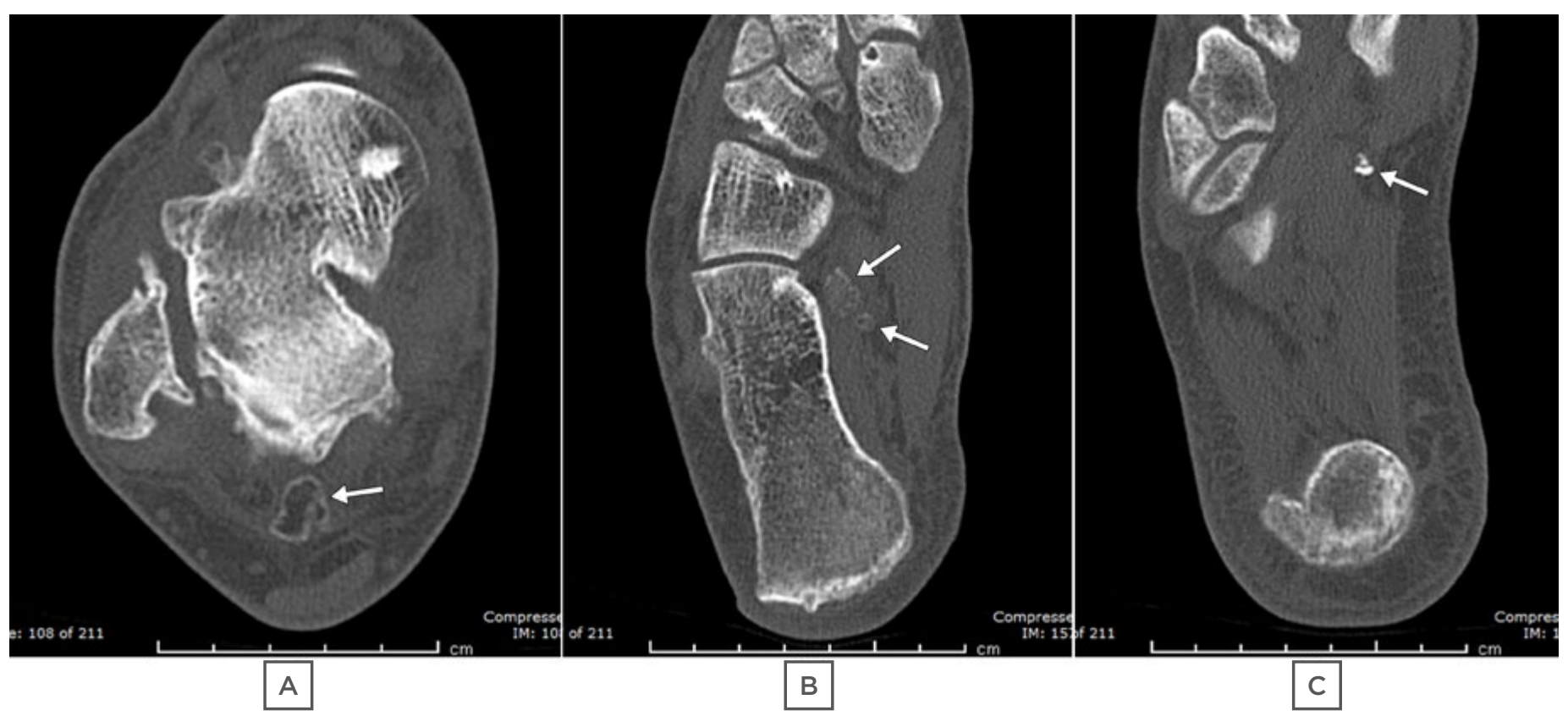

Figure 1: A 51-year-old male with ankle stiffness, swelling, and previous ankle sprain, requiring immobilisation in plaster 10 years previously.

A), B) and C) show CTs of the right ankle, demonstrating loose bodies (arrows) in Zones 1, 2, and 3, respectively. 


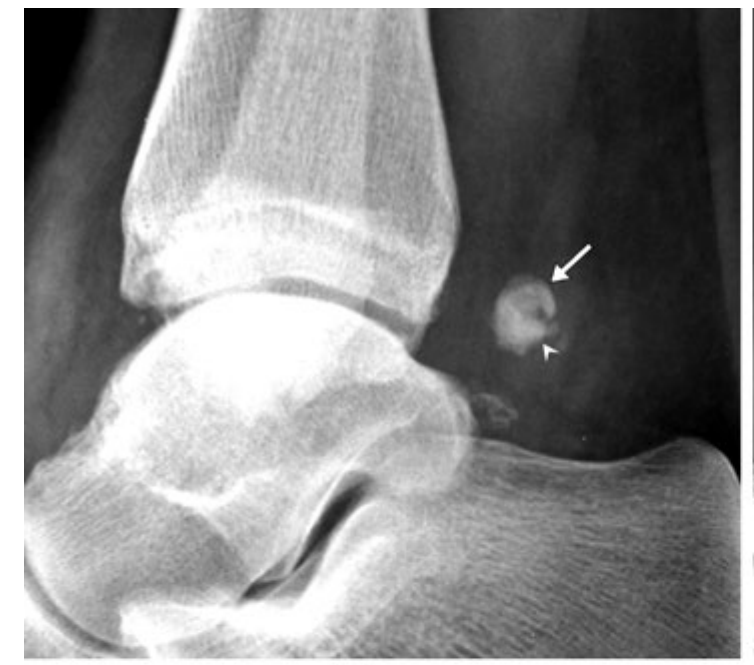

A

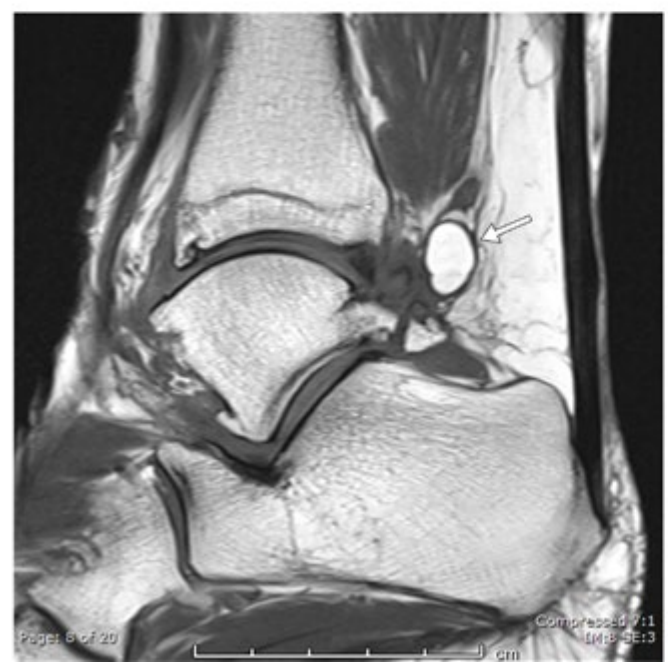

c

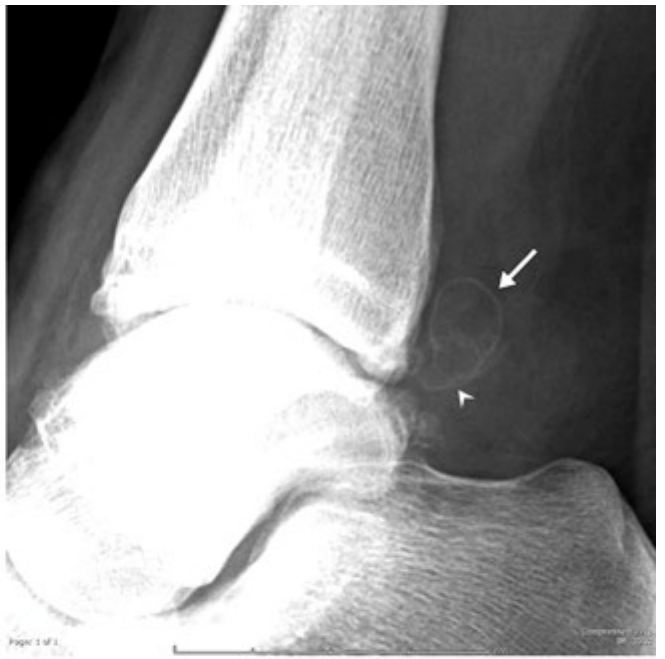

B

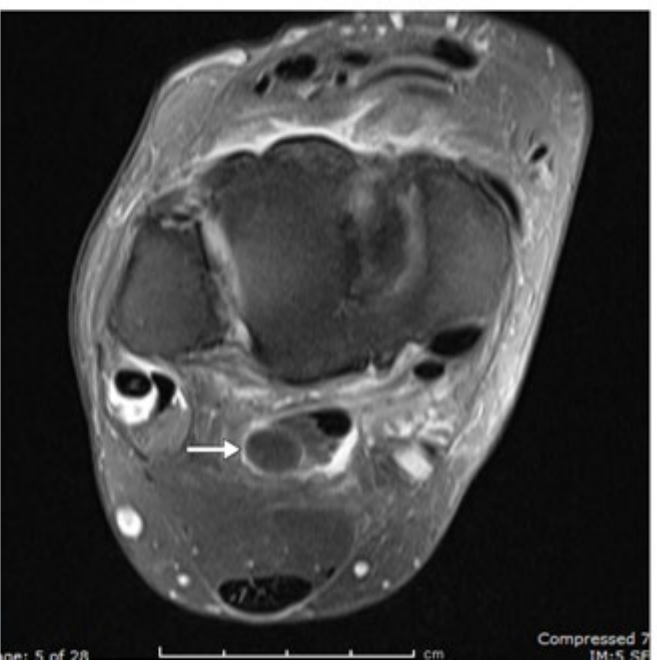

D

\section{Figure 2: A 60-year-old female with increasing ankle pain.}

A) and B): lateral radiographs taken 24 months apart, showing initial dense appearance of two overlapping loose bodies (arrow and arrowhead) in image $\mathbf{A}$ ), which altered to a radiolucent appearance with a sclerotic rim in $\mathbf{B}$ ). Note slight increase in size. C) T1 sagittal and D) T1 TIRM are sagittal images demonstrating the larger loose body to have high marrow fat content.

TIRM: turbo inversion recovery magnitude.

All patients in this study had radiological evidence of osteoarthritic changes in their ankle joint. Of note, a large proportion of patients (17/34 cases; 50\%) either progressed to, or already had surgical fusion or replacement of their ankle joints. Nineteen of the cases also had suffered traumatic injuries to their ankles in the past. The authors believe these loose bodies have formed in the ankle joints from their osteoarthritis or trauma, which subsequently migrated into the $\mathrm{FHL}$ tendon sheath.
Interval imaging, where available, has also been reviewed. This revealed a few possible outcomes for the loose bodies. Firstly, the loose bodies may gradually change from a trabeculated to lucent appearance whilst maintaining a sclerotic rim. This is in keeping with fatty marrow replacement, reflecting maturation. This change is often accompanied by an increase in size (Figure 2). Secondly, loose bodies can also increase in size whilst maintaining their trabeculated pattern, with the maximal growth of $4 \mathrm{~mm}$ measured 
in a patient (most proximal loose body; Figure 3). Third and finally, the loose bodies can demonstrate gradual reduction in sizes and radiodensities (Figure 4), suggesting gradual resorption. Resorption of a loose body is only possible with synovial attachment to allow intra-synovial resorption. ${ }^{8}$

Synovial attachment would explain the relative static appearances of the loose bodies in this patient despite them becoming smaller, which theoretically should have increased their propensity to become more mobile and relocate along the length of the whole tendon sheath. The presence of revascularisation, a requirement for ossification, ${ }^{9}$ also provides another explanation as to why these loose bodies had remained not only in the same Zones, but roughly in the same position. In addition, just as myositis ossificans can resolve due to mechanical motion from muscle activity, the authors think loose bodies in the tendon sheath can also resolve via the same mechanism due to motion from the adjacent tendon.

The MR appearances of intra-articular loose bodies have been well-described in the literature ${ }^{10,11}$ and can have variable signal, depending on the fat content and degree of calcification, differing between chondral, osteochondral, and osseous loose bodies. In the authors' series, 88 loose bodies were imaged on MRI; 24 demonstrated fatty marrow signal of high $\mathrm{T1}$, low on fat-suppressed fluid sensitive sequences; 63 demonstrated low signal on both $\mathrm{T} 1$ and fat-suppressed fluid sensitive sequences; while 1 loose body had low T1 and intermediate to low signal on fat-suppressed, fluid-sensitive sequences (but non-ossified on $\mathrm{CT}$ ), in keeping with a cartilaginous loose body.

There is a tendency for larger loose bodies to be seen in Zone 1 rather than Zone 2, in line with

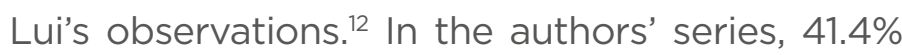
of Zone 1 loose bodies measured $8 \mathrm{~mm}$ or more compared with just $15.4 \%$ of Zone 2 loose bodies. The authors suspect that larger loose bodies would be prevented from distal migration due to the limiting space in the tendon sheath. Some of the larger Zone 2 loose bodies were also noted to be aligned longitudinally with their largest dimension along the length of the FHL tendon sheath. The authors postulate that these loose bodies were of smaller sizes when they first migrated into Zone 2, where they subsequently grew in size with maximal growth along the longitudinal length of the FHL tendon sheath. In other words, the authors think the $\mathrm{FHL}$ tendon sheath may have influenced their growth to the longitudinal dimension.

As far as the authors' know, there has only been one reported case of migratory loose bodies into the FHL tendon sheath. ${ }^{2}$ It is important to distinguish this entity from other differential diagnoses such as primary tenosynovial chondromatosis of the tendon sheath where the ankle joint is normal ${ }^{12,13}$ or myositis ossificans, where the ossification is intramuscular rather than in the tendon sheath. ${ }^{14}$ Primary chondromatosis is conventionally described as a metaplastic process, though a cytogenetic study has proposed it to be a neoplastic process with chondral proliferation of the synovium;"15 it has a rare potential for malignant transformation..$^{16}$ Its aetiology is distinctly different from secondary chondromatosis, which is a sequela of osteochondral injuries. Furthermore, an important facet in the management of loose bodies, in general, is to determine and treat their underlying source; this is equally if not more important than just surgically removing the loose bodies. ${ }^{17}$ It is, therefore, important to appreciate this migration phenomenon so as not to overlook an ankle pathology such as advanced osteoarthritis ${ }^{18}$.

Determining the exact location of a loose body, whether it lies in the posterior recess of the ankle joint or in the FHL tendon sheath, can be difficult. This distinction is important ${ }^{12}$ as access to the FHL tendon sheath loose bodies will require the endoscopist to surgically release the covering fascia. ${ }^{18}$

A loose body with high fatty content may be misdiagnosed as a lipomatous lesion on MRI, or even missed altogether on fat-suppressed or inversion recovery sequences (Figure 2). This can be prevented by correlating an MRI study with previous radiograph or $\mathrm{CT}$ examinations and, indeed, it should always be conducted when available. In addition, the authors found fat-suppressed sequences to be unhelpful, especially in the case of the spiculated loose body (Figure 5). However, interpretation of the loose body is straightforward if correlated with either radiograph or CT. Nevertheless, MRI is superior in detecting non-mineralised loose bodies. 


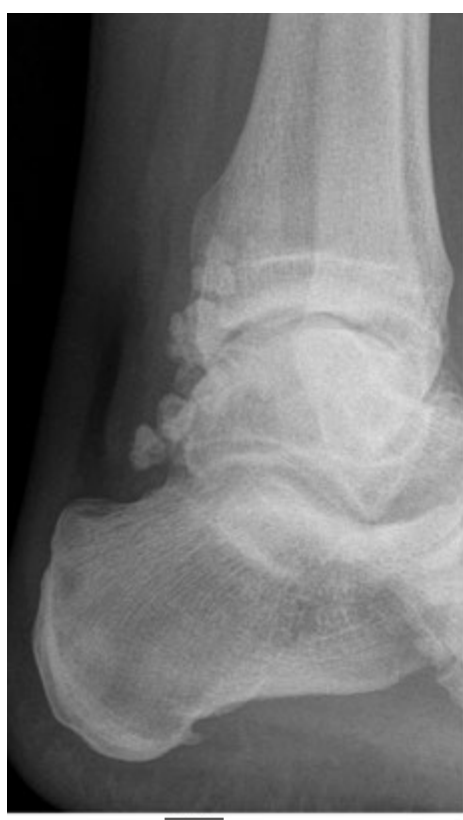

A

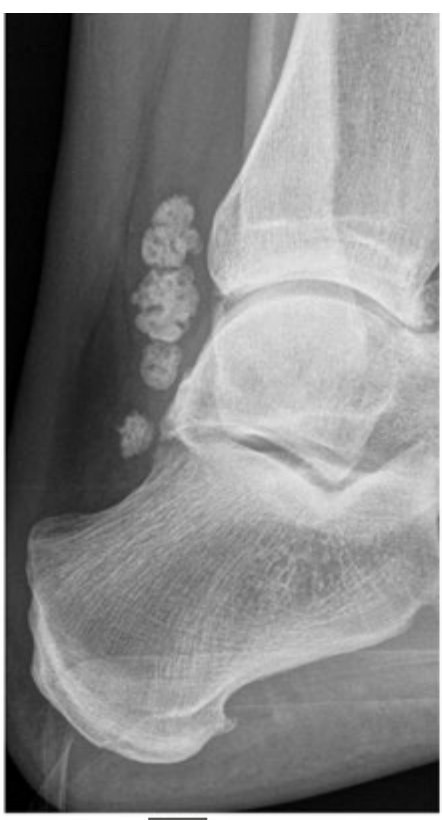

B

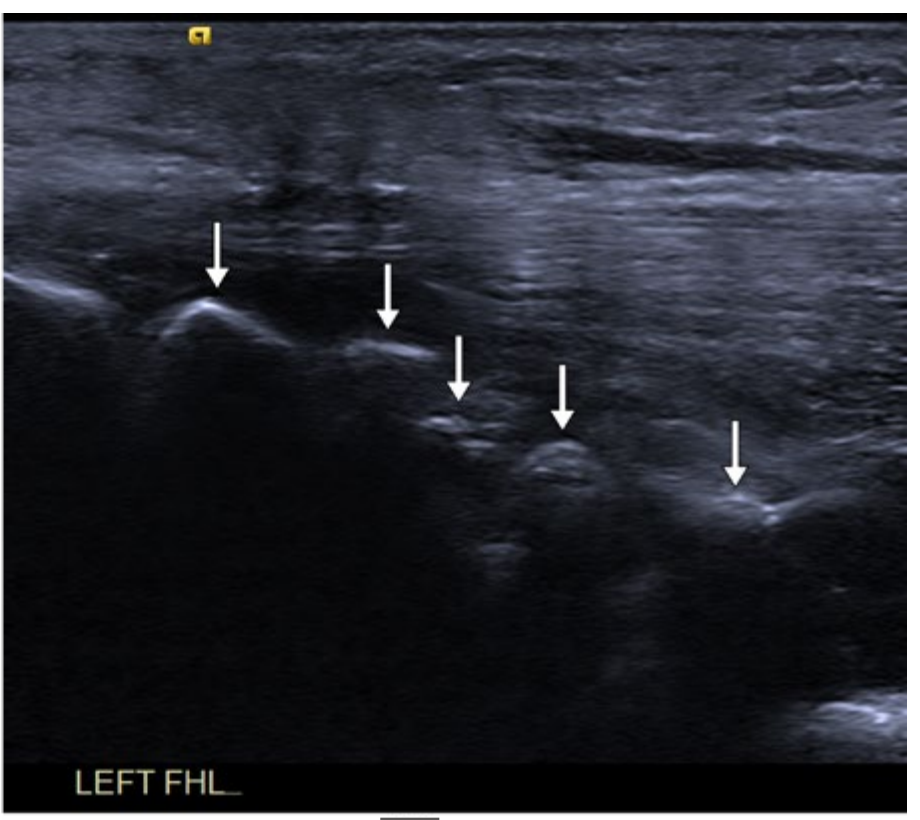

c

Figure 3: A 47-year-old male with peroneal brevis tenosynovitis associated with a large ganglion (not shown).

A) and B): lateral radiographs taken 41 months apart demonstrating multiple ossified loose bodies in Zone 1 of the FHL tendon sheath. These can also be seen on ultrasound (arrows) in image $\mathbf{C}$ ), which is a longitudinal image across Zone 1 of the FHL tendon. The patient was asymptomatic from these loose bodies.

FHL: flexor hallucis longus.

Ultrasound can also be utilised to visualise loose bodies, revealing focal areas of high reflectivity with posterior acoustic shadowing indicating presence of peripheral mineralisation (Figure $3 \mathrm{C}$ ). Cartilaginous loose bodies, on the other hand, may demonstrate hypoechogenic structures, with a central area of hyperechogenicity reflecting internal calcification..$^{19}$ Understandably, detection of loose bodies using ultrasound is aided by the presence of surrounding fluid. ${ }^{20}$ The authors' experience has shown that ultrasound of these loose bodies can demonstrate a degree of limited mobility.

The authors' report constitutes the largest series of loose bodies in the FHL tendon sheath having migrated from the ankle joint to date. The strength of this study is that all their patients had either cross-sectional studies (CT or MRI) or an ultrasound examination, where the relationship of the loose bodies within the FHL tendon sheath can be determined with confidence. On MRI, the loose bodies must be surrounded by tenosynovial fluid, or be seen to efface the edge of the tendon or myotendinous junction. In addition, care was taken during review of CT to ensure it was not intra-tendinous calcification mimicking loose bodies in the tendon sheath; this is especially relevant in the context of previous trauma. In the presence of any ambiguity, the location of the calcification was assessed using multiplanar reconstruction. Indeed, as reported above, one case of CT has been removed due to inconclusive imaging appearance.

The authors noticed that there is a disproportionate high number of male patients (25 male and 8 female) and of the right ankle (21 right and 13 left) in their series. This can be partially explained by the fact that there were 6 male patients with football-related injuries. There were no female football-related injuries. In addition, right-footed dominance is more common in the general population. ${ }^{21}$

There are two weaknesses in this study. Firstly, the authors do not have histological data on the loose bodies, which would have been able to definitively differentiate between primary and secondary synovial chondromatosis. A proportion of these patients had surgery to their ankle joints, but the FHL tendon sheath loose bodies had not 


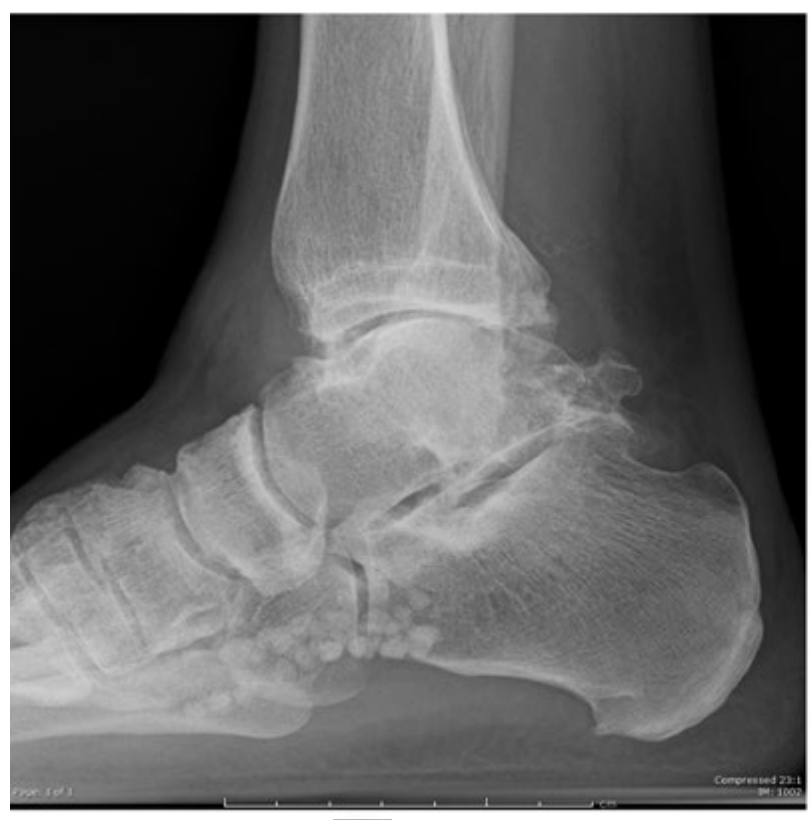

A

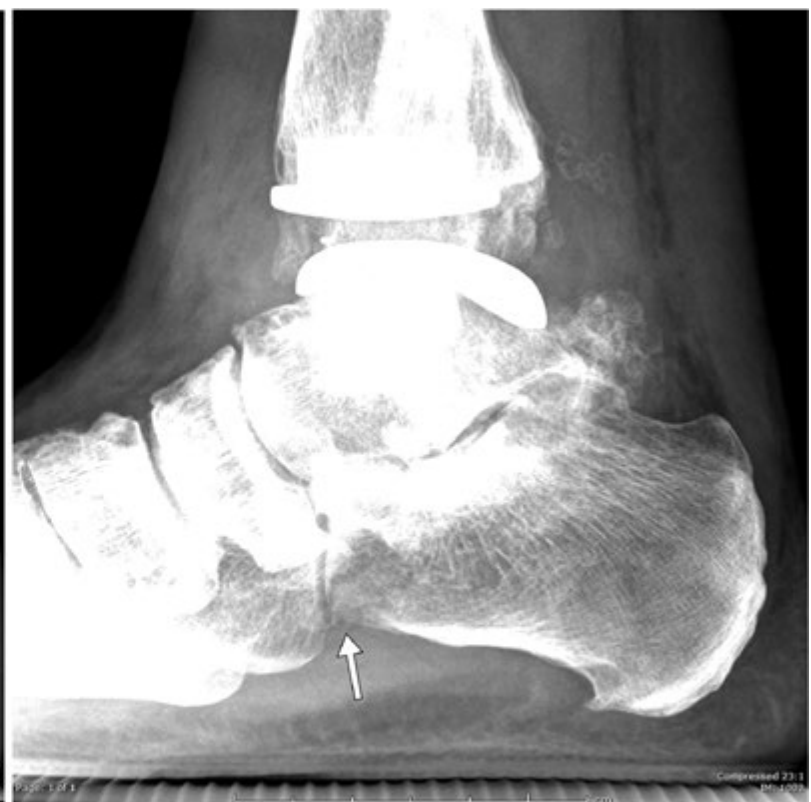

B

Figure 4: A 65-year-old male with progressive right ankle pain, limiting his activities.

There are multiple loose bodies in the FHL tendon sheath, confirmed on MRI (not shown). A) and B) are serial radiographs of the right ankle, demonstrating severe ankle osteoarthritis in $\mathbf{A}$ ), progressing later to ankle replacement. B) Multiple of the Zone 2 loose bodies have been resorbed 6 months after ankle replacement, with a few remaining visualised as centrally lucent, peripherally sclerotic loose bodies (arrow).

FHL: flexor hallucis longus.

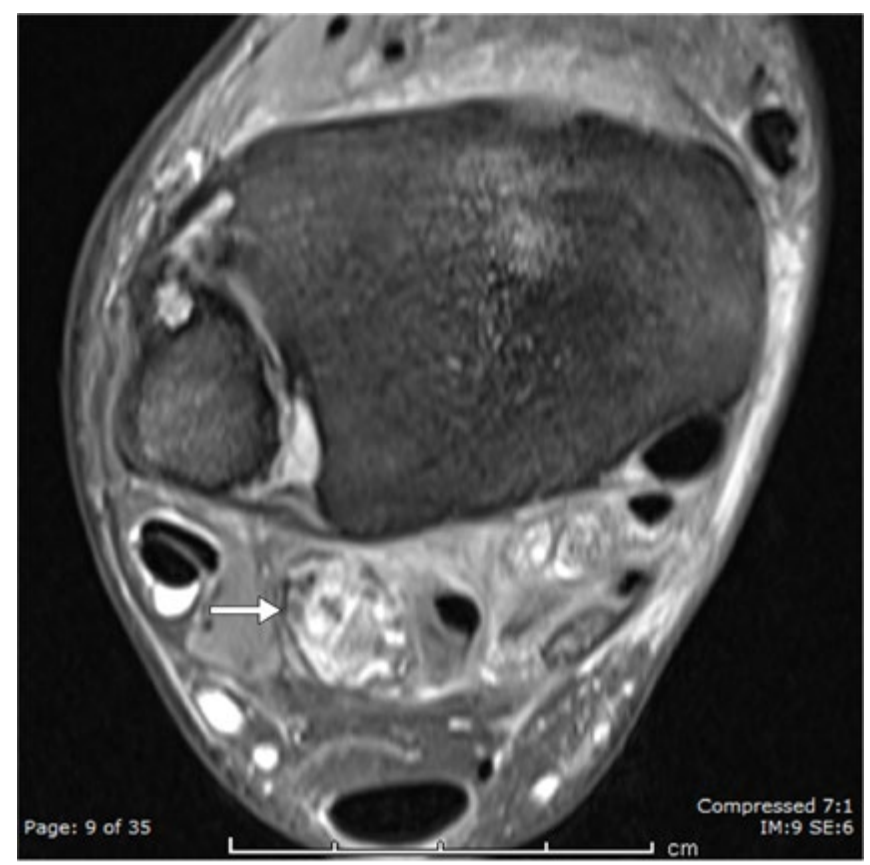

Figure 5: A 52-year-old male with progressive ankle pain, swelling, and osteoarthritis.

PD FS axial image demonstrating a spiculated loose body (arrow), which is difficult to interpret in isolation without a plain radiograph or CT (performed, but not shown here).

PD FS: proton density with fat saturation. 
been specifically addressed as they were not the cause of the patients' symptoms. Nevertheless, there are no imaging features supporting primary tenosynovial chondromatosis in this cohort. Tenosynovial chondromatosis lesions are either fusiform, round, or oval, and these features are not present in this cohort. ${ }^{22}$ Furthermore, chondroid synovial proliferation, which is a recognised feature of primary synovial chondromatosis, ${ }^{23}$ is not present in the authors' cases.

Secondly, the authors only have radiological confirmation of communication between ankle joint and FHL tendon sheath in five cases, by way of gadolinium confirmation or presence of large contiguous effusion on MRI. Literature has reported the presence of communication between the ankle joint and the $\mathrm{FHL}$ tendon sheath to be around $17 \%,{ }^{\prime}$ but it would have been ideal to confirm this communication radiologically by using, for example, iodinated contrast under fluoroscopy screening. However, this would have required an invasive procedure, which could not have been clinically justified.

\section{CONCLUSION}

In summary, the authors' study represents the largest series showing loose bodies in the FHL tendon sheath having migrated from the ankle joint to date. Osteochondral insults in the ankle joint may produce loose bodies that can migrate into the FHL tendon sheath. All the patients in this study demonstrated osteoarthritis in their ankle joints, with a significant portion with advanced osteoarthritic changes and ankle instability. Presence of loose bodies in the ankle joint should prompt closer inspection of the ankle joint for any articular disease. Finally, it is important to recognise migratory loose bodies in the $\mathrm{FHL}$ tendon sheath as an entity distinctively different from primary tenosynovial chondromatosis as it may influence surgical management and clinical outcome.

\section{References}

1. Na JB et al. The flexor hallucis longus: tenographic technique and correlation of imaging findings with surgery in 39 ankles. Radiology. 2005;236(3):974-82.

2. Shah A et al. Flexor hallucis longus loose bodies- an unusual cause of plantar midfoot pain. OMCIS J Radiology. 2013;2(8):1000152.

3. Lui TH. Flexor hallucis longus tendoscopy: a technical note. Knee Surg Sports Traumatol Arthrosc. 2009;17(1):107-10.

4. Froelich JM, Hillard-Sembell D. Symptomatic loose bodies of the knee located in a popliteal cyst. Orthopedics. 2009;32(12):918.

5. Kim SH et al. Idiopathic synovial osteochondromatosis of the hip: radiographic and $M R$ appearances in 15 patients. Korean J Radiol. 2002;3(4):254-9.

6. Lunn JV et al. Arthroscopic synovectomy, removal of loose bodies and selective biceps tenodesis for synovial chondromatosis of the shoulder. J Bone Joint Surg Br. 2007;89(10):1329-35.

7. Ko JY et al. Synovial chondromatosis of the subacromial bursa with rotator cuff tearing. J Shoulder Elbow Surg. 1995;4(4):312-6.

8. Milgram JW et al. Multiple loose bodies: formation, revascularization, and resorption. A 29-year followup study. Clin Orthop Relat Res. 1996;(322):152-7.

9. Saotome $\mathrm{K}$ et al. Histologic classification of loose bodies in osteoarthrosis. J Orthop Sci. 2006;11(6):607-13.

10. Brossmann $\mathrm{J}$ et al. Imaging of osseous and cartilaginous intraarticular bodies in the knee: comparison of MR imaging and MR arthrography with CT and CT arthrography in cadavers. Radiology. 1996;200(2):509-17.

11. Murphey MD et al. Imaging of synovial chondromatosis with radiologic-pathologic correlation. Radiographics. 2007;27(5):1465-88.

12. Lui TH. Tenosynovial osteochondromatosis of the flexor hallucis longus tendon treated by tendoscopy. J Foot Ankle Surg. 2015;54(4):758-64.

13. Oakley $\mathrm{J}$ et al. Tenosynovial osteochondromatosis of the flexor hallucis longus tendon. Foot Ankle Surg. 2010;16(3):148-50

14. Clint $\mathrm{S}$ et al. Posterior ankle pain due to myositis ossificans of flexor hallucis longus - a case report. Foot and Ankle Surgery. 2003;9(2):137-40.

15. Buddingh EP et al. Chromosome 6 abnormalities are recurrent in synovial chondromatosis. Cancer Genet Cytogenet. 2003;140(1):18-22.
16. Davis Rl et al. Primary synovial chondromatosis: a clinicopathologic review and assessment of malignant potential. Hum Pathol. 1998;29(7):683-8.

17. Dave $\mathrm{OH}$ et al. Arthroscopic management of elbow plica and loose bodies. Morrey BF et al (eds.), Morrey's The Elbow and Its Disorders (2018) $5^{\text {th }}$ edition, Philadelphia: Elsevier, pp.194-9.

18. Lui TH. Endoscopic removal of loose bodies of the posterior ankle extra-articular space arising from flexor hallucis longus tenosynovial osteochondromatosis. Arthrosc Tech. 2016;5(6):e1247-52.

19. Court-Payen M. Sonography of the knee: Intra-articular pathology. J Clin Ultrasound. 2004;32(9):481-90.

20. Frankel DA et al. Synovial joints: evaluation of intraarticular bodies with US. Radiology. 1998;206(1):41-4.

21. Porac C, Coren S. Lateral Preferences and Human Behavior (1981) New York: Springer-Verlag.

22. Walker EA et al. Imaging characteristics of tenosynovial and bursal chondromatosis. Skeletal Radiol. 2011;40(3):317-25.

23. Milgram JW. Synovial osteochondromatosis: a histopathological study of thirty cases. J Bone Joint Surg Am. 1977;59(6):792-801. 